\title{
Nonlinear Super Integrable Couplings of Super Broer-Kaup-Kupershmidt Hierarchy and Its Super Hamiltonian Structures
}

\author{
Sixing $\mathrm{Tao}^{1}$ and Tiecheng $\mathrm{Xia}^{2}$ \\ ${ }^{1}$ School of Mathematics and Information Science, Shangqiu Normal University, \\ Shangqiu 476000, China \\ ${ }^{2}$ Department of Mathematics, Shanghai University, Shanghai 200444, China
}

Correspondence should be addressed to Sixing Tao; taosixing@163.com

Received 25 February 2013; Revised 26 July 2013; Accepted 9 August 2013

Academic Editor: Xing Biao Hu

Copyright (C) 2013 S. Tao and T. Xia. This is an open access article distributed under the Creative Commons Attribution License, which permits unrestricted use, distribution, and reproduction in any medium, provided the original work is properly cited.

\begin{abstract}
Nonlinear integrable couplings of super Broer-Kaup-Kupershmidt hierarchy based upon an enlarged matrix Lie super algebra were constructed. Then its super Hamiltonian structures were established by using super trace identity, and the conserved functionals were proved to be in involution in pairs under the defined Poisson bracket. As its reduction, nonlinear integrable couplings of the classical integrable hierarchy were obtained.
\end{abstract}

\section{Introduction}

With the development of soliton theory, super integrable systems associated with Lie super algebra have aroused growing attentions by many mathematicians and physicists. It was known that super integrable systems contained the odd variables, which would provide more prolific fields for mathematical researchers and physical ones. Several super integrable systems, including super AKNS hierarchy, super KdV hierarchy, super NLS-MKdV hierarchy, super Tu hierarchy, super Broer-Kaup-Kupershmidt hierarchy, have been studied in $[1-8]$. There are some interesting results on the super integrable systems, such as Darboux transformation in [9], super Hamiltonian structures in [10-12] binary nonlinearization in [13], and reciprocal transformation in [14].

The research of integrable couplings of the well-known integrable hierarchy has received considerable attention in [15-23] A few approaches to construct linear integrable couplings of the classical soliton equation are presented by permutation, enlarging spectral problem, using matrix Lie algebra [24] constructing new loop Lie algebra and creating semidirect sums of Lie algebra. Recently, Ma [25] and Ma and $\mathrm{Zhu}$ [26] presented a scheme for constructing nonlinear continuous and discrete integrable couplings using the block type matrix algebra. However, there is one interesting question for us is how to generate nonlinear super integrable couplings for the super integrable hierarchy.

In this paper, we would like to construct nonlinear super integrable couplings of the super soliton equations through enlarging matrix Lie super algebra. We take the Lie algebra $B(0,1)$ as an example to illustrate the approach for extending Lie super algebras. Based on the enlarged Lie super algebra $g l(6,2)$, we work out nonlinear super integrable Hamiltonian couplings of the super Broer-Kaup-Kupershmidt hierarchy. Finally, we will reduce the nonlinear super Broer-KaupKupershmidt integrable Hamiltonian couplings to some special cases.

\section{Enlargement of Lie Super Algebra $B(0,1)$}

Consider the Lie super algebra $B(0,1)$. Its basis is

$$
E_{1}=\left(\begin{array}{ccc}
1 & 0 & 0 \\
0 & -1 & 0 \\
0 & 0 & 0
\end{array}\right), \quad E_{2}=\left(\begin{array}{lll}
0 & 1 & 0 \\
0 & 0 & 0 \\
0 & 0 & 0
\end{array}\right),
$$




$$
\begin{gathered}
E_{3}=\left(\begin{array}{lll}
0 & 0 & 0 \\
1 & 0 & 0 \\
0 & 0 & 0
\end{array}\right), \quad E_{4}=\left(\begin{array}{ccc}
0 & 0 & 1 \\
0 & 0 & 0 \\
0 & -1 & 0
\end{array}\right), \\
E_{5}=\left(\begin{array}{lll}
0 & 0 & 0 \\
0 & 0 & 1 \\
1 & 0 & 0
\end{array}\right),
\end{gathered}
$$

where $E_{1}, E_{2}$, and $E_{3}$ are even elements and $E_{4}$, and $E_{5}$ are odd elements. Their nonzero (anti)commutation relations are

$$
\begin{array}{lll}
{\left[E_{1}, E_{2}\right]=2 E_{2},} & {\left[E_{1}, E_{3}\right]=-2 E_{3},} & {\left[E_{1}, E_{4}\right]=E_{4},} \\
{\left[E_{1}, E_{5}\right]=-E_{5},} & {\left[E_{2}, E_{3}\right]=E_{1},} & {\left[E_{2}, E_{5}\right]=E_{4},} \\
{\left[E_{3}, E_{4}\right]=E_{5},} & {\left[E_{4}, E_{4}\right]=-2 E_{2},} & {\left[E_{4}, E_{5}\right]=E_{1},} \\
{\left[E_{5}, E_{5}\right]=2 E_{3} .} &
\end{array}
$$

Let us enlarged the Lie super algebra $B(0,1)$ to the Lie super algebra $g l(6,2)$ with a basis

$$
\begin{aligned}
& e_{1}=\left(\begin{array}{ccccc}
1 & 0 & 0 & 0 & 0 \\
0 & -1 & 0 & 0 & 0 \\
0 & 0 & 1 & 0 & 0 \\
0 & 0 & 0 & -1 & 0 \\
0 & 0 & 0 & 0 & 0
\end{array}\right), \quad e_{2}=\left(\begin{array}{lllll}
0 & 1 & 0 & 0 & 0 \\
0 & 0 & 0 & 0 & 0 \\
0 & 0 & 0 & 1 & 0 \\
0 & 0 & 0 & 0 & 0 \\
0 & 0 & 0 & 0 & 0
\end{array}\right), \\
& e_{3}=\left(\begin{array}{ccccc}
0 & 0 & 0 & 0 & 0 \\
1 & 0 & 0 & 0 & 0 \\
0 & 0 & 0 & 0 & 0 \\
0 & 0 & 1 & 0 & 0 \\
0 & 0 & 0 & 0 & 0
\end{array}\right), \quad e_{4}=\left(\begin{array}{ccccc}
0 & 0 & 1 & 0 & 0 \\
0 & 0 & 0 & -1 & 0 \\
0 & 0 & 1 & 0 & 0 \\
0 & 0 & 0 & -1 & 0 \\
0 & 0 & 0 & 0 & 0
\end{array}\right), \\
& e_{5}=\left(\begin{array}{ccccc}
0 & 0 & 0 & 1 & 0 \\
0 & 0 & 0 & 0 & 0 \\
0 & 0 & 0 & 1 & 0 \\
0 & 0 & 0 & 0 & 0 \\
0 & 0 & 0 & 0 & 0
\end{array}\right), \quad e_{6}=\left(\begin{array}{lllll}
0 & 0 & 0 & 0 & 0 \\
0 & 0 & 1 & 0 & 0 \\
0 & 0 & 0 & 0 & 0 \\
0 & 0 & 1 & 0 & 0 \\
0 & 0 & 0 & 0 & 0
\end{array}\right), \\
& e_{7}=\left(\begin{array}{ccccc}
0 & 0 & 0 & 0 & 1 \\
0 & 0 & 0 & 0 & 0 \\
0 & 0 & 0 & 0 & 0 \\
0 & 0 & 0 & 0 & 0 \\
0 & -1 & 0 & 1 & 0
\end{array}\right), \quad e_{8}=\left(\begin{array}{ccccc}
0 & 0 & 0 & 0 & 0 \\
0 & 0 & 0 & 0 & 1 \\
0 & 0 & 0 & 0 & 0 \\
0 & 0 & 0 & 0 & 0 \\
1 & 0 & -1 & 0 & 0
\end{array}\right) \text {, }
\end{aligned}
$$

where $e_{1}, e_{2}, e_{3}, e_{4}, e_{5}$, and $e_{6}$ are even, and $e_{7}$, and $e_{8}$ are odd.

The generator of Lie super algebra $g l(6,2), e_{i}, 0 \leq i \leq 8$, satisfy the following (anti)commutation relations:

$$
\begin{array}{llll}
{\left[e_{1}, e_{2}\right]=2 e_{2},} & {\left[e_{1}, e_{3}\right]=-2 e_{3},} & {\left[e_{1}, e_{5}\right]=2 e_{5},} \\
{\left[e_{1}, e_{6}\right]=-2 e_{6},} & {\left[e_{1}, e_{7}\right]=e_{7},} & {\left[e_{1}, e_{8}\right]=-e_{8},} \\
{\left[e_{2}, e_{3}\right]=e_{1},} & {\left[e_{2}, e_{4}\right]=-2 e_{5},} & {\left[e_{2}, e_{6}\right]=e_{4},} \\
{\left[e_{2}, e_{8}\right]=e_{7},} & {\left[e_{3}, e_{4}\right]=2 e_{6},} & {\left[e_{3}, e_{5}\right]=-e_{4},}
\end{array}
$$

$$
\begin{aligned}
{\left[e_{3}, e_{7}\right] } & =e_{8}, \quad\left[e_{4}, e_{5}\right]=2 e_{5}, \quad\left[e_{4}, e_{6}\right]=-2 e_{6}, \\
{\left[e_{5}, e_{6}\right] } & =e_{4}, \quad\left[e_{7}, e_{8}\right]=e_{1}-e_{4}, \quad\left[e_{7}, e_{7}\right]=2 e_{5}-2 e_{2}, \\
{\left[e_{8}, e_{8}\right] } & =2 e_{3}-2 e_{6}, \\
{\left[e_{1}, e_{4}\right] } & =\left[e_{2}, e_{5}\right]=\left[e_{2}, e_{7}\right]=\left[e_{3}, e_{6}\right]=\left[e_{3}, e_{8}\right] \\
& =\left[e_{4}, e_{7}\right]=\left[e_{4}, e_{8}\right]=\left[e_{5}, e_{7}\right]=\left[e_{5}, e_{8}\right] \\
& =\left[e_{6}, e_{7}\right]=\left[e_{6}, e_{8}\right]=0 .
\end{aligned}
$$

Define a loop super algebra corresponding to the Lie superalgebra $g l(6,2)$, denote by

$$
\begin{aligned}
\tilde{g} l(6,2)= & g l(6,2) \otimes \mathbb{C}\left[\lambda, \lambda^{-1}\right] \\
= & \left\{e_{i} \lambda^{m}, e_{i} \in g l(6,2), i=1,2, \ldots, 8,\right. \\
& m=0, \pm 1, \pm 2, \ldots\} .
\end{aligned}
$$

The corresponding (anti)commutative relations are given as

$$
\left[e_{i} \lambda^{m}, e_{j} \lambda^{n}\right]=\left[e_{i}, e_{j}\right] \lambda^{m+n}, \quad \forall e_{i}, e_{j} \in g l(6,2) .
$$

\section{Nonlinear Super Integrable Couplings of Super Broer-Kaup-Kupershmidt Hierarchy}

Let us start from an enlarged spectral problem associated with $g l(6,2)$ :

$$
\begin{gathered}
\phi_{x}=U(u, \lambda) \phi, \\
U=e_{1}(1)+r e_{1}(0)+s e_{2}(0)+e_{3}(0) \\
+u_{1} e_{4}(0)+u_{2} e_{5}(0)+\alpha e_{7}(0)+\beta e_{8}(0) \\
=\left(\begin{array}{ccccc}
\lambda+r & s & u_{1} & u_{2} & \alpha \\
1 & -\lambda-r & 0 & -u_{1} & \beta \\
0 & 0 & \lambda+r+u_{1} & s+u_{2} & 0 \\
0 & 0 & 1 & -\lambda-r-u_{1} & 0 \\
\beta & -\alpha & -\beta & \alpha & 0
\end{array}\right),
\end{gathered}
$$

where $r, s, u_{1}$, and $u_{2}$ are even potentials but $\alpha$ and $\beta$ are odd ones.

In ordr to obtain super integrable couplings of super integrable hierarchy, we first solve the adjoint representation of (7),

$$
V_{x}=[U, V]
$$

with

$$
\begin{aligned}
V= & A e_{1}(0)+B e_{2}(0)+C e_{3}(0)+E e_{4}(0) \\
& +F e_{5}(0)+G e_{6}(0)+\rho e_{7}(0)+\delta e_{8}(0) \\
& \left(\begin{array}{ccccc}
A & B & E & F & \rho \\
C & -A & G & -E & \delta \\
0 & 0 & A+E & B+F & 0 \\
0 & 0 & C+G & -A-E & 0 \\
\delta & -\rho & -\delta & \rho & 0
\end{array}\right)
\end{aligned}
$$


where $A, B, C, E, F$, and $G$ are commuting fields and $\rho$, and $\delta$ are anticommuting fields. Then we obtain

$$
\begin{gathered}
A_{x}=-B+s C+\beta \rho+\alpha \delta, \\
B_{x}=-2 s A+2 \lambda B+2 r B-2 \alpha \rho, \\
C_{x}=2 A-2 \lambda C-2 r C+2 \beta \delta, \\
E_{x}=u_{2} C-F+s G+u_{2} G-\alpha \delta-\beta \rho, \\
F_{x}=-2 u_{2} A+2 u_{1} B-2 s E \\
-2 u_{2} E+2 \lambda F+2 r F+2 u_{1} F+2 \alpha \rho, \\
G_{x}=-2 u_{1} C+2 E-2 \lambda G-2 r G-2 u_{1} G-2 \beta \delta, \\
\rho_{x}=-\alpha A-\beta B+\lambda \rho+r \rho+s \delta, \\
\delta_{x}=\beta A-\alpha C+\rho-\lambda \delta-r \delta .
\end{gathered}
$$

Substituting

$$
\begin{aligned}
A & =\sum_{m \geq 0} A_{m} \lambda^{-m}, & B & =\sum_{m \geq 0} B_{m} \lambda^{-m}, \\
C & =\sum_{m \geq 0} C_{m} \lambda^{-m}, & E & =\sum_{m \geq 0} E_{m} \lambda^{-m}, \\
F & =\sum_{m \geq 0} F_{m} \lambda^{-m}, & G & =\sum_{m \geq 0} G_{m} \lambda^{-m}, \\
\rho & =\sum_{m \geq 0} \rho_{m} \lambda^{-m}, & \delta & =\sum_{m \geq 0} \delta_{m} \lambda^{-m},
\end{aligned}
$$

into the previous equation gives the following recursive formulas:

$$
\begin{aligned}
A_{m, x}= & -B_{m}+s C_{m}+\beta \rho_{m}+\alpha \delta_{m}, \\
B_{m, x}= & -2 s A_{m}+2 B_{m+1}+2 r B_{m}-2 \alpha \rho_{m}, \\
C_{m, x}= & 2 A_{m}-2 C_{m+1}-2 r C_{m}+2 \beta \delta_{m}, \\
E_{m, x}= & u_{2} C_{m}-F_{m}+s G_{m}+u_{2} G_{m}-\alpha \delta_{m}-\beta \rho_{m}, \\
F_{m, x}= & -2 u_{2} A_{m}+2 u_{1} B_{m}-2 s E_{m} \\
& -2 u_{2} E_{m}+2 F_{m+1}+2 r F_{m}+2 u_{1} F_{m}+2 \alpha \rho_{m}, \\
G_{m, x}= & -2 u_{1} C_{m}+2 E_{m}-2 G_{m+1}-2 r G_{m}-2 u_{1} G_{m}-2 \beta \delta_{m}, \\
\rho_{m, x}= & -\alpha A_{m}-\beta B_{m}+\rho_{m+1}+r \rho_{m}+s \delta_{m}, \\
\delta_{m, x}= & \beta A_{m}-\alpha C_{m}+\rho_{m}-\delta_{m+1}-r \delta_{m} .
\end{aligned}
$$

From these equations, we can successively deduce

$$
\begin{aligned}
& A_{0}=1, \quad B_{0}=C_{0}=F_{0}=G_{0}=\rho_{0}=\delta_{0}=0, \\
& E_{0}=\varepsilon=\text { constant }, \quad A_{1}=0, \quad B_{1}=s, \quad C_{1}=1, \\
& E_{1}=0, \quad F_{1}=u_{2}+\varepsilon u_{2}+\varepsilon s, \quad G_{1}=\varepsilon,
\end{aligned}
$$

$$
\begin{aligned}
\rho_{1}= & \alpha, \quad \delta_{1}=\beta, \quad A_{2}=-\frac{1}{2} s-\alpha \beta, \\
B_{2}= & \frac{1}{2} s_{x}-r s, \quad C_{2}=-r, \\
E_{2}= & -\frac{1}{2} u_{2}-\frac{1}{2} \varepsilon u_{2}-\frac{1}{2} \varepsilon s+\alpha \beta, \\
F_{2}= & \frac{1}{2} u_{2 x}+\frac{1}{2} \varepsilon u_{2 x}+\frac{1}{2} \varepsilon s_{x}-s u_{1} \\
& -r u_{2}-\varepsilon r u_{2}-\varepsilon r s-u_{1} u_{2}-\varepsilon u_{1} u_{2}-\varepsilon s u_{1}, \\
G_{2}= & -u_{1}-\varepsilon u_{1}-\varepsilon r, \quad \rho_{2}=\alpha_{x}-r \alpha, \\
\delta_{2}= & -\beta_{x}-r \beta, \quad A_{3}=-\frac{1}{4} s_{x}+r s+\alpha \beta_{x}-\alpha_{x} \beta+2 r \alpha \beta, \\
B_{3}= & \frac{1}{4} s_{x x}-\frac{1}{2} r_{x} s-r s_{x}-\frac{1}{2} s^{2}-s \alpha \beta+r^{2} s+\alpha \alpha_{x}, \\
C_{3}= & \frac{1}{2} r_{x}-\frac{1}{2} s-\alpha \beta+r^{2}-\beta \beta_{x}, \\
E_{3}= & (\varepsilon+1)\left(r u_{2}+s u_{1}+u_{1} u_{2}-\frac{1}{4} u_{2 x}\right)+\varepsilon r s-\frac{1}{4} \varepsilon s_{x} \\
& -\alpha \beta_{x}+\alpha_{x} \beta-2 r \alpha \beta, \\
F_{3}= & (\varepsilon+1) \\
& \times\left(-\frac{1}{2} s u_{1 x}-s_{x} u_{1}-\frac{1}{2} r_{x} u_{2}-r u_{2 x}-\frac{1}{2} u_{1 x} u_{2}\right. \\
\delta_{3}= & \beta_{x x}+r_{x} \beta+2 r \beta_{x}-\frac{1}{2} s \beta+\alpha_{x}+r^{2} \beta . \\
& +s \alpha \beta+\frac{1}{4} \varepsilon s_{x x}-\frac{1}{2} \varepsilon s^{2} \\
& -\varepsilon r s_{x}-\frac{1}{2} \varepsilon r_{x} s+\varepsilon r_{1}^{2} s-\alpha \alpha_{x}, \\
G_{3}= & \frac{1}{2} u_{1 x}+\frac{1}{2} \varepsilon u_{1 x}+\frac{1}{2} \varepsilon r_{x}+2 r u_{1}-\frac{1}{2} u_{2} \\
& -\frac{1}{2} \varepsilon u_{2}-\frac{1}{2} \varepsilon s+\alpha \beta+2 \varepsilon r u_{1}+\varepsilon r^{2}+u_{1}^{2}+\varepsilon u_{1}^{2}+\beta \beta_{x}, \\
& \quad s u_{2}-\frac{1}{2} u_{2}^{2}+r^{2} u_{2}
\end{aligned}
$$

Equations (12) can be written as

$$
\left(\begin{array}{c}
4 A_{m+1}+2 E_{m+1} \\
2 C_{m+1}+G_{m+1} \\
2 A_{m+1}+2 E_{m+1} \\
C_{m+1}+G_{m+1} \\
2 \delta_{m+1} \\
-2 \rho_{m+1}
\end{array}\right)=L\left(\begin{array}{c}
4 A_{m}+2 E_{m} \\
2 C_{m}+G_{m} \\
2 A_{m}+2 E_{m} \\
C_{m}+G_{m} \\
2 \delta_{m} \\
-2 \rho_{m}
\end{array}\right)
$$

where 


$$
L=\left(\begin{array}{cccccc}
\frac{1}{2} \partial-\partial^{-1} r \partial & -s-\partial^{-1} s \partial & -\partial^{-1} u_{1} \partial & -u_{2}-\partial^{-1} u_{2} \partial & -\frac{1}{2} \alpha-\partial^{-1} \alpha \partial & \frac{1}{2} \beta-\partial^{-1} \beta \partial \\
\frac{1}{2} & -\frac{1}{2} \partial-r & 0 & -2 u_{1} & \frac{1}{2} \beta & 0 \\
0 & 0 & L_{33} & L_{34} & 0 & 0 \\
0 & 0 & \frac{1}{2} & -\frac{1}{2} \partial-r-u_{1} & 0 & 0 \\
\beta & -2 \alpha & -\beta & \alpha & -\partial-r & -1 \\
-\alpha+\beta \partial & -2 s \beta & \alpha-\beta \partial & 2 s \beta & s+\alpha \beta & \partial-r
\end{array}\right)
$$

with

$$
\begin{aligned}
& L_{33}=\frac{1}{2} \partial-\partial^{-1} r \partial-\partial^{-1} u_{1} \partial, \\
& L_{34}=-s-u_{2}-\partial^{-1} s \partial-\partial^{-1} u_{2} \partial .
\end{aligned}
$$

Then, let us consider the spectral problem (7) with the following auxiliary spectral problem:

$$
\phi_{t_{n}}=V^{(n)} \phi,
$$

with

$$
\begin{aligned}
V^{(n)}= & \sum_{j=0}^{n}\left(\begin{array}{ccccc}
A_{j} & B_{j} & E_{j} & F_{j} & \rho_{j} \\
C_{j} & -A_{j} & G_{j} & -E_{j} & \delta_{j} \\
0 & 0 & A_{j}+E_{j} & B_{j}+F_{j} & 0 \\
0 & 0 & C_{j}+G_{j} & -A_{j}-E_{j} & 0 \\
\delta_{j} & -\rho_{j} & -\delta_{j} & \rho_{j} & 0
\end{array}\right) \lambda^{n-j} \\
& +\left(\begin{array}{ccccc}
-C_{n+1} & 0 & -G_{n+1} & 0 & 0 \\
0 & C_{n+1} & 0 & G_{n+1} & 0 \\
0 & 0 & -C_{n+1}-G_{n+1} & 0 & 0 \\
0 & 0 & 0 & C_{n+1}+G_{n+1} & 0 \\
0 & 0 & 0 & 0 & 0
\end{array}\right) .
\end{aligned}
$$

From the compatible condition, $\phi_{x, t_{n}}=\phi_{t_{n}, x}$, according to (7) and (17), we get the zero curvature equation:

$$
U_{t_{n}}-V_{x}^{(n)}+\left[U, V^{(n)}\right]=0,
$$

which gives a nonlinear Lax super integrable hierarchy:

$$
\begin{aligned}
u_{t_{n}} & =\left(\begin{array}{c}
r \\
s \\
u_{1} \\
u_{2} \\
\alpha \\
\beta
\end{array}\right)_{t_{n}} \\
= & \left(\begin{array}{c}
-C_{n+1, x} \\
2 B_{n+1}-2 s C_{n+1} \\
-G_{n+1, x} \\
-2 u_{2} C_{n+1}+2 F_{n+1}-2 s G_{n+1}-2 u_{2} G_{n+1} \\
\rho_{n+1}-\alpha C_{n+1} \\
-\delta_{n+1}+\beta C_{n+1}
\end{array}\right) .
\end{aligned}
$$

The super integrable hierarchy (20) is a nonlinear super integrable couplings for the super BKK hierarchy:

$$
\widetilde{u}_{t_{n}}=\left(\begin{array}{c}
r \\
s \\
\alpha \\
\beta
\end{array}\right)_{t_{n}}=\left(\begin{array}{c}
-C_{n+1, x} \\
2 B_{n+1}-2 s C_{n+1} \\
\rho_{n+1}-\alpha C_{n+1} \\
-\delta_{n+1}+\beta C_{n+1}
\end{array}\right) .
$$

\section{Super Hamiltonian Structures}

A direct calculation reads

$$
\begin{array}{ll}
\operatorname{Str}\left(U_{\lambda}, V\right)=4 A+2 E, & \operatorname{Str}\left(U_{r}, V\right)=4 A+2 E, \\
\operatorname{Str}\left(U_{s}, V\right)=2 C+G, & \operatorname{Str}\left(U_{u_{1}}, V\right)=C+G, \\
\operatorname{Str}\left(U_{u_{2}}, V\right)=B+F, & \operatorname{Str}\left(U_{\alpha}, V\right)=2 \delta, \\
\operatorname{Str}\left(U_{\beta}, V\right)=-2 \rho . &
\end{array}
$$

Substituting the above results into the super trace identity in $[11,12]$,

$$
\frac{\delta}{\delta u} \int \operatorname{Str}\left(\frac{\delta U}{\delta \lambda} V\right) \mathrm{d} x=\lambda^{-\gamma} \frac{\partial}{\partial \lambda} \lambda^{\gamma} \operatorname{Str}\left(\frac{\delta U}{\delta u} V\right)
$$

yields that

$$
\frac{\delta}{\delta u} \int(4 A+2 E) \mathrm{d} x=\lambda^{-\gamma} \frac{\partial}{\partial \lambda} \lambda^{\gamma}\left(\begin{array}{c}
4 A+2 E \\
2 C+G \\
2 A+2 E \\
C+G \\
2 \delta \\
-2 \rho
\end{array}\right) .
$$

Comparing the coefficients of $\lambda^{-n-1}$ on both side of (24),

$$
\frac{\delta}{\delta u} \int\left(4 A_{n+1}+2 E_{n+1}\right) \mathrm{d} x=\lambda^{-\gamma} \frac{\partial}{\partial \lambda} \lambda^{\gamma}\left(\begin{array}{c}
4 A_{n}+2 E_{n} \\
2 C_{n}+G_{n} \\
2 A_{n}+2 E_{n} \\
C_{n}+G_{n} \\
2 \delta_{n} \\
-2 \rho_{n}
\end{array}\right),
$$


From the initial values in (12), we obtain $\gamma=0$. Thus we have

$$
\begin{aligned}
& \frac{\mathscr{H}_{n}}{\delta u}=\left(\begin{array}{c}
4 A_{n}+2 E_{n} \\
2 C_{n}+G_{n} \\
2 A_{n}+2 E_{n} \\
C_{n}+G_{n} \\
2 \delta_{n} \\
-2 \rho_{n}
\end{array}\right), \\
& \mathscr{H}_{n}=-\left(\frac{1}{n+1}\left(4 A_{n+1}+2 E_{n+1}\right) \mathrm{d} x, \quad n \geq 0 .\right.
\end{aligned}
$$

It then follows that the nonlinear super integrable couplings (20) possess the following super Hamiltonian form

$$
u_{t_{n}}=K_{n}(u)=J \frac{\delta \mathscr{H}_{n}}{\delta u},
$$

where

$$
J=\left(\begin{array}{cccccc}
0 & -\partial & 0 & \partial & 0 & 0 \\
-\partial & 0 & \partial & 0 & \alpha & -\beta \\
0 & \partial & 0 & -2 \partial & 0 & 0 \\
\partial & 0 & -2 \partial & 0 & -\alpha & \beta \\
0 & -\alpha & 0 & \alpha & 0 & -\frac{1}{2} \\
0 & \beta & 0 & -\beta & -\frac{1}{2} & 0
\end{array}\right)
$$

is a super Hamiltonian operator and $\mathscr{H}_{n}(n \geq 0)$ are Hamiltonian functions.

It can be verified that

$$
J^{*}=\left(\begin{array}{cccccc}
0 & \partial & 0 & -\partial & 0 & 0 \\
\partial & 0 & -\partial & 0 & -\alpha & \beta \\
0 & -\partial & 0 & 2 \partial & 0 & 0 \\
-\partial & 0 & 2 \partial & 0 & \alpha & -\beta \\
0 & \alpha & 0 & -\alpha & 0 & -\frac{1}{2} \\
0 & -\beta & 0 & \beta & -\frac{1}{2} & 0
\end{array}\right), \quad J^{*} L=L^{*} J^{*}
$$

where $J^{*}$ and $L^{*}$ are conjugate operators of $J$ and $L$, respectively.

If we define the following Poisson bracket

$$
\left[\mathscr{H}_{n}, \mathscr{H}_{m}\right]=\left(\frac{\delta \mathscr{H}_{n}}{\delta u}, J \frac{\delta \mathscr{H}_{m}}{\delta u}\right)
$$

$$
=\int\left(\frac{\delta \mathscr{H}_{n}}{\delta r}, \frac{\delta \mathscr{H}_{n}}{\delta s}, \frac{\delta \mathscr{H}_{n}}{\delta u_{1}}, \frac{\delta \mathscr{H}_{n}}{\delta u_{2}}, \frac{\delta \mathscr{H}_{n}}{\delta \alpha}, \frac{\delta \mathscr{H}_{n}}{\delta \beta}\right)
$$

$$
\times\left(\begin{array}{c}
\frac{\delta \mathscr{H}_{m}}{\delta r} \\
\frac{\delta \mathscr{H}_{m}}{\delta s} \\
\frac{\delta \mathscr{H}_{m}}{\delta u_{1}} \\
\frac{\delta \mathscr{H}_{m}}{\delta u_{2}} \\
\frac{\delta \mathscr{H}_{m}}{\delta \alpha} \\
\frac{\delta \mathscr{H}_{m}}{\delta \beta}
\end{array}\right) \mathrm{d} x,
$$

where $(\cdot, \cdot)$ denotes the inner product, as $\left(\delta \mathscr{H}_{n} / \delta \alpha\right)\left(\delta \mathscr{H}_{m} /\right.$ $\delta \beta)=-\left(\delta \mathscr{H}_{m} / \delta \beta\right)\left(\delta \mathscr{H}_{n} / \delta \alpha\right)$, we can obtain

$$
\begin{aligned}
{\left[\mathscr{H}_{n}, \mathscr{H}_{m}\right]=\int\left(\frac{\delta \mathscr{H}_{m}}{\delta r}, \frac{\delta \mathscr{H}_{m}}{\delta s}, \frac{\delta \mathscr{H}_{m}}{\delta u_{1}}, \frac{\delta \mathscr{H}_{m}}{\delta u_{2}}, \frac{\delta \mathscr{H}_{m}}{\delta \alpha}, \frac{\delta \mathscr{H}_{m}}{\delta \beta}\right) } \\
\times J^{*}\left(\begin{array}{c}
\frac{\delta \mathscr{H}_{n}}{\delta r} \\
\frac{\delta \mathscr{H}_{n}}{\delta s} \\
\frac{\delta \mathscr{H}_{n}}{\delta u_{1}} \\
\frac{\delta \mathscr{H}_{n}}{\delta u_{2}} \\
\frac{\delta \mathscr{H}_{n}}{\delta \alpha} \\
\frac{\delta \mathscr{H}_{n}}{\delta \beta}
\end{array}\right) \mathrm{d} x=-\left[\mathscr{H}_{m}, \mathscr{H}_{n}\right] .
\end{aligned}
$$

From (14) and (31), we have

$$
\frac{\delta \mathscr{H}_{m+1}}{\delta u}=L \frac{\delta \mathscr{H}_{m}}{\delta u}, \quad \frac{\delta \mathscr{H}_{m}}{\delta u}=L^{m} \frac{\delta \mathscr{H}_{0}}{\delta u} .
$$

Suppose $k, l$ are arbitrary nonnegative integers consider

$$
\left[\mathscr{H}_{k}, \mathscr{H}_{k+l}\right]=\left(L^{k} \frac{\delta \mathscr{H}_{0}}{\delta u}, J L^{k+l} \frac{\delta \mathscr{H}_{0}}{\delta u}\right) .
$$


By using the properties of $J$ and $L$ in (29), we can obtain

$$
\begin{aligned}
{\left[\mathscr{H}_{k}, \mathscr{H}_{k+l}\right] } \\
=\left(L^{k} \frac{\delta \mathscr{H}_{0}}{\delta u}, J L^{k+l} \frac{\delta \mathscr{H}_{0}}{\delta u}\right)=\left(L^{k} \frac{\delta \mathscr{H}_{0}}{\delta u},(J L) L^{k+l-1} \frac{\delta \mathscr{H}_{0}}{\delta u}\right) \\
=\left((J L)^{*} L^{k} \frac{\delta \mathscr{H}_{0}}{\delta u}, L^{k+l-1} \frac{\delta \mathscr{H}_{0}}{\delta u}\right) \\
=\left(L^{*} J^{*} L^{k} \frac{\delta \mathscr{H}_{0}}{\delta u}, L^{k+l-1} \frac{\delta \mathscr{H}_{0}}{\delta u}\right) \\
=\left(J^{*} L^{k+1} \frac{\delta \mathscr{H}_{0}}{\delta u}, L^{k+l-1} \frac{\delta \mathscr{H}_{0}}{\delta u}\right) \\
=\left(L^{k+1} \frac{\delta \mathscr{H}_{0}}{\delta u}, J L^{k+l-1} \frac{\delta \mathscr{H}_{0}}{\delta u}\right) \\
=\left(\frac{\delta \mathscr{H}_{k+1}}{\delta u}, J \frac{\delta \mathscr{H}_{k+l-1}}{\delta u}\right)=\left[\mathscr{H}_{k+1}, \mathscr{H}_{k+l-1}\right] .
\end{aligned}
$$

Ascending the subscript of left factor $\mathscr{H}_{k}$, then we have

$$
\left[\mathscr{H}_{k}, \mathscr{H}_{k+l}\right]=\left[\mathscr{H}_{k+l}, \mathscr{H}_{k}\right]
$$

By using the antisymmetric law of Poisson bracket (31), we have

$$
\left[\mathscr{H}_{k}, \mathscr{H}_{k+l}\right]=0
$$

So the conserved functionals $\left\{\mathscr{H}_{n}\right\}_{1}^{\infty}$ are in involution in pairs under the Poisson bracket (30).

\section{Reductions}

Taking $\alpha=\beta=0$, the hierarchy (27) reduces to a nonlinear integrable couplings of the Broer-Kaup-Kupershmidt hierarchy.

When $n=2$ in (27), we obtain the nonlinear super couplings of the second super Broer-Kaup-Kupershmidt equations:

$$
\begin{gathered}
r_{t_{2}}=-\frac{1}{2} r_{x x}-2 r r_{x}+\frac{1}{2} s_{x}+\alpha_{x} \beta+\alpha \beta_{x}+\beta \beta_{x x}, \\
s_{t_{2}}=\frac{1}{2} s_{x x}-2 r_{x} s-2 r s_{x}+2 \alpha \alpha_{x}+2 s \beta \beta_{x}, \\
u_{1, t_{2}}=(\varepsilon+1)\left(-\frac{1}{2} u_{1 x x}-2 r_{x} u_{1}-2 r u_{1 x}+\frac{1}{2} u_{2 x}\right. \\
\left.-2 u_{1} u_{1 x}-\frac{1}{2} r_{x x}+\frac{1}{2} s_{x}-2 r r_{x}\right) \\
+\frac{1}{2} r_{x x}-\frac{1}{2} s_{x}-\alpha_{x} \beta-\alpha \beta_{x}+2 r r_{x}-\beta \beta_{x x},
\end{gathered}
$$

$$
\begin{gathered}
u_{2, t_{2}}=(\varepsilon+1)\left(\frac{1}{2} u_{2 x x}-2 s_{x} u_{1}-2 s u_{1 x}-2 r_{x} u_{2}\right. \\
-2 r u_{2 x}-2 u_{1 x} u_{2}-2 u_{1} u_{2 x} \\
\left.+\frac{1}{2} s_{x x}-2 r_{x} s-2 r s_{x}\right) \\
-\frac{1}{2} s_{x x}+2 r_{x} s+2 r s_{x}-2 \alpha \alpha_{x}-2 s \beta \beta_{x}, \\
\alpha_{t_{2}}=\alpha_{x x}-2 r \alpha_{x}-\frac{3}{2} r_{x} \alpha+\frac{1}{2} s_{x} \beta+s \beta_{x}+\alpha \beta \beta_{x}, \\
\beta_{t_{2}}=-\beta_{x x}-\frac{1}{2} r_{x} \beta-2 r \beta_{x}-\alpha_{x} .
\end{gathered}
$$

Particularly, taking $\alpha=\beta=0$ in (37), we can get the nonlinear integrable couplings of the second order Broer-KaupKupershmidt equations:

$$
\begin{aligned}
& r_{t_{2}}=-\frac{1}{2} r_{x x}-2 r r_{x}+\frac{1}{2} s_{x} \\
& s_{t_{2}}=\frac{1}{2} s_{x x}-2 r_{x} s-2 r s_{x} \text {, } \\
& u_{1, t_{2}}=(\varepsilon+1)\left(-\frac{1}{2} u_{1 x x}-2 r_{x} u_{1}-2 r u_{1 x}+\frac{1}{2} u_{2 x}\right. \\
& \left.-2 u_{1} u_{1 x}-\frac{1}{2} r_{x x}+\frac{1}{2} s_{x}-2 r r_{x}\right) \\
& +\frac{1}{2} r_{x x}-\frac{1}{2} s_{x}+2 r r_{x} \\
& u_{2, t_{2}}=(\varepsilon+1) \\
& \times\left(\frac{1}{2} u_{2 x x}-2 s_{x} u_{1}-2 s u_{1 x}\right. \\
& -2 r_{x} u_{2}-2 r u_{2 x}-2 u_{1 x} u_{2}-2 u_{1} u_{2 x} \\
& \left.+\frac{1}{2} s_{x x}-2 r_{x} s-2 r s_{x}\right) \\
& -\frac{1}{2} s_{x x}+2 r_{x} s+2 r s_{x}
\end{aligned}
$$

Let $\varepsilon=0$ in (37); we have

$$
\begin{gathered}
r_{t_{2}}=-\frac{1}{2} r_{x x}-2 r r_{x}+\frac{1}{2} s_{x}+\alpha_{x} \beta+\alpha \beta_{x}+\beta \beta_{x x}, \\
s_{t_{2}}=\frac{1}{2} s_{x x}-2 r_{x} s-2 r s_{x}+2 \alpha \alpha_{x}+2 s \beta \beta_{x}, \\
u_{1, t_{2}}=-\frac{1}{2} u_{1 x x}-2 r_{x} u_{1}-2 r u_{1 x}+\frac{1}{2} u_{2 x}-2 u_{1} u_{1 x} \\
\quad-\alpha_{x} \beta-\alpha \beta_{x}-\beta \beta_{x x}, \\
u_{2, t_{2}}=\frac{1}{2} u_{2 x x}-2 s_{x} u_{1}-2 s u_{1 x}-2 r_{x} u_{2}-2 r u_{2 x} \\
\quad-2 u_{1 x} u_{2}-2 u_{1} u_{2 x}-2 \alpha \alpha_{x}-2 s \beta \beta_{x},
\end{gathered}
$$




$$
\begin{aligned}
& \alpha_{t_{2}}=\alpha_{x x}-2 r \alpha_{x}-\frac{3}{2} r_{x} \alpha+\frac{1}{2} s_{x} \beta+s \beta_{x}+\alpha \beta \beta_{x}, \\
& \beta_{t_{2}}=-\beta_{x x}-\frac{1}{2} r_{x} \beta-2 r \beta_{x}-\alpha_{x} .
\end{aligned}
$$

If setting $\varepsilon=-1, u_{1}=-r$, and $u_{2}=-s$ in (37), we obtain the second order super Broer-Kaup-Kupershmidt equations:

$$
\begin{aligned}
& r_{t_{2}}=-\frac{1}{2} r_{x x}-2 r r_{x}+\frac{1}{2} s_{x}+\alpha_{x} \beta+\alpha \beta_{x}+\beta \beta_{x x}, \\
& s_{t_{2}}=\frac{1}{2} s_{x x}-2 r_{x} s-2 r s_{x}+2 \alpha \alpha_{x}+2 s \beta \beta_{x}, \\
& \alpha_{t_{2}}=\alpha_{x x}-2 r \alpha_{x}-\frac{3}{2} r_{x} \alpha+\frac{1}{2} s_{x} \beta+s \beta_{x}+\alpha \beta \beta_{x}, \\
& \beta_{t_{2}}=-\beta_{x x}-\frac{1}{2} r_{x} \beta-2 r \beta_{x}-\alpha_{x} .
\end{aligned}
$$

\section{Remarks}

In this paper, we introduced an approach for constructing nonlinear integrable couplings of super integrable hierarchy. Zhang [27] once employed two kinds of explicit Lie algebra $F$ and $G$ to obtain the nonlinear integrable couplings of the GJ hierarchy and Yang hierarchy, respectively. It is easy to see that Lie algebra $F$ given in [27] is isomorphic to the Lie algebra span $\left\{e_{1}, e_{2}, e_{3}, e_{4}, e_{5}, e_{6}\right\}$ in $g l(6,2)$. So we can obtain nonlinear integrable couplings of super GJ and Yang hierarchy easily. The method in this paper can be applied to other super integrable systems for constructing their integrable couplings.

\section{Acknowledgments}

This work was supported by the National Natural Science Foundation of China (no. 61072147), the Natural Science Foundation of Henan Province (no. 132300410202), the Science and Technology Key Research Foundation of the Education Department of Henan Province (no. 12A110017) and the Youth Research Foundation of Shangqiu Normal University (no. 2011QN12).

\section{References}

[1] B. A. Kupershmidt, "Odd and even Poisson brackets in dynamical systems," Letters in Mathematical Physics, vol. 9, no. 4, pp. 323-330, 1985.

[2] Y. S. Li and L. N. Zhang, "A note on the super AKNS equations," Journal of Physics, vol. 21, no. 7, pp. 1549-1552, 1988.

[3] M.-H. Tu and J.-C. Shaw, "Hamiltonian structures of generalized Manin-Radul super-KdV and constrained super KP hierarchies," Journal of Mathematical Physics, vol. 40, no. 6, pp. 3021-3034, 1999.

[4] Q. P. Liu and X.-B. Hu, "Bilinearization of $N=1$ supersymmetric Korteweg-de Vries equation revisited," Journal of Physics, vol. 38, no. 28, pp. 6371-6378, 2005.
[5] H.-H. Dong and X. Wang, "Lie algebras and Lie super algebra for the integrable couplings of NLS-MKdV hierarchy," Communications in Nonlinear Science and Numerical Simulation, vol. 14, no. 12, pp. 4071-4077, 2009.

[6] X.-Z. Wang and X.-K. Liu, "Two types of Lie super-algebra for the super-integrable Tu-hierarchy and its super-Hamiltonian structure," Communications in Nonlinear Science and Numerical Simulation, vol. 15, no. 8, pp. 2044-2049, 2010.

[7] H.-X. Yang and Y.-P. Sun, "Hamiltonian and super-Hamiltonian extensions related to Broer-Kaup-Kupershmidt system," International Journal of Theoretical Physics, vol. 49, no. 2, pp. 349364, 2010.

[8] Q. P. Liu, X.-B. Hu, and M.-X. Zhang, "Supersymmetric modified Korteweg-de Vries equation: bilinear approach," Nonlinearity, vol. 18, no. 4, pp. 1597-1603, 2005.

[9] H. Aratyn, E. Nissimov, and S. Pacheva, "Supersymmetric Kadomtsev-Petviashvili hierarchy: "ghost" symmetry structure, reductions, and Darboux-Bäcklund solutions," Journal of Mathematical Physics, vol. 40, no. 6, pp. 2922-2932, 1999.

[10] C. Morosi and L. Pizzocchero, "On the bi-Hamiltonian structure of the supersymmetric KdV hierarchies. A Lie superalgebraic approach," Communications in Mathematical Physics, vol. 158 , no. 2, pp. 267-288, 1993.

[11] X.-B. Hu, "An approach to generate superextensions of integrable systems," Journal of Physics, vol. 30, no. 2, pp. 619-632, 1997.

[12] W.-X. Ma, J.-S. He, and Z.-Y. Qin, "A supertrace identity and its applications to superintegrable systems," Journal of Mathematical Physics, vol. 49, no. 3, Article ID 033511, 2008.

[13] J. He, J. Yu, Y. Cheng, and R. Zhou, "Binary nonlinearization of the super AKNS system," Modern Physics Letters B, vol. 22, no. 4, pp. 275-288, 2008.

[14] Q. P. Liu, Z. Popowicz, and K. Tian, "Supersymmetric reciprocal transformation and its applications," Journal of Mathematical Physics, vol. 51, no. 9, Article ID 093511, 2010.

[15] W.-X. Ma, "Enlarging spectral problems to construct integrable couplings of soliton equations," Physics Letters A, vol. 316, no. 1-2, pp. 72-76, 2003.

[16] W.-X. Ma, X.-X. Xu, and Y. Zhang, "Semidirect sums of Lie algebras and discrete integrable couplings," Journal of Mathematical Physics, vol. 47, no. 5, Article ID 053501, 2006.

[17] W.-X. Ma, X.-X. Xu, and Y. Zhang, "Semi-direct sums of Lie algebras and continuous integrable couplings," Physics Letters A, vol. 351, no. 3, pp. 125-130, 2006.

[18] F. Guo and Y. Zhang, "The quadratic-form identity for constructing the Hamiltonian structure of integrable systems," Journal of Physics, vol. 38, no. 40, pp. 8537-8548, 2005.

[19] W.-X. Ma and M. Chen, "Hamiltonian and quasi-Hamiltonian structures associated with semi-direct sums of Lie algebras," Journal of Physics, vol. 39, no. 34, pp. 10787-10801, 2006.

[20] T.-C. Xia and F.-C. You, "Generalized multi-component TC hierarchy and its multi-component integrable coupling system," Communications in Theoretical Physics, vol. 44, no. 5, pp. 793798, 2005.

[21] T.-C. Xia and F.-C. You, "Multi-component Dirac equations hierarchy and its multicomponent integrable couplings system," Chinese Physics, vol. 16, pp. 605-610, 2007.

[22] Y. Zhang and E. Fan, "Coupling integrable couplings and biHamiltonian structure associated with the Boiti-Pempinelli-Tu hierarchy," Journal of Mathematical Physics, vol. 51, no. 8, Article ID 083506, 2010. 
[23] Y. Zhang and H. Tam, "Four Lie algebras associated with $R^{6}$ and their applications," Journal of Mathematical Physics, vol. 51, no. 9, Article ID 093514, 2010.

[24] Y. Zhang and H. Zhang, "A direct method for integrable couplings of TD hierarchy," Journal of Mathematical Physics, vol. 43, no. 1, pp. 466-472, 2002.

[25] W.-X. Ma, "Nonlinear continuous integrable Hamiltonian couplings," Applied Mathematics and Computation, vol. 217, no. 17, pp. 7238-7244, 2011.

[26] W.-X. Ma and Z.-N. Zhu, "Constructing nonlinear discrete integrable Hamiltonian couplings," Computers \& Mathematics with Applications, vol. 60, no. 9, pp. 2601-2608, 2010.

[27] Y.-F. Zhang, "Lie algebras for constructing nonlinear integrable couplings," Communications in Theoretical Physics, vol. 56, no. 5, pp. 805-812, 2011. 


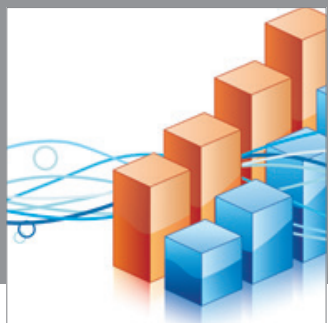

Advances in

Operations Research

mansans

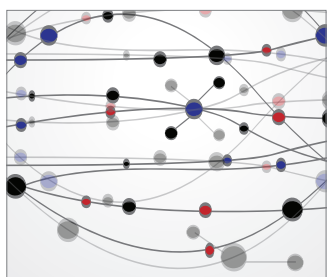

The Scientific World Journal
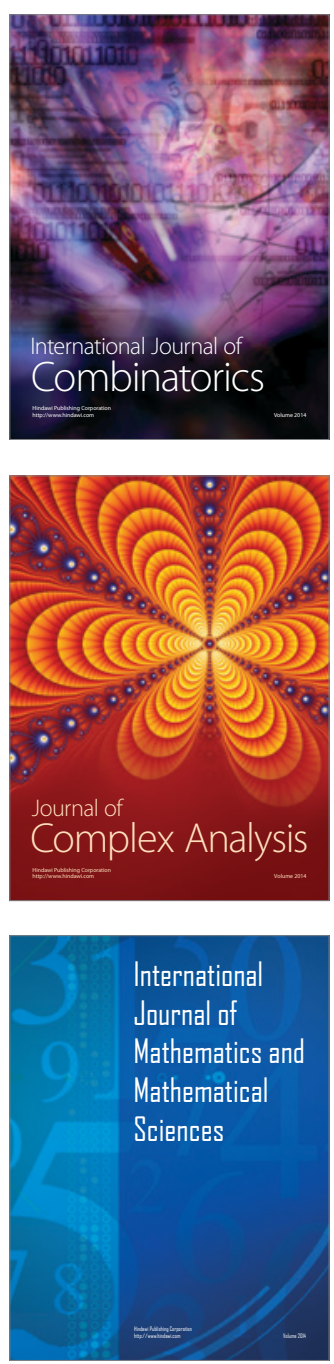
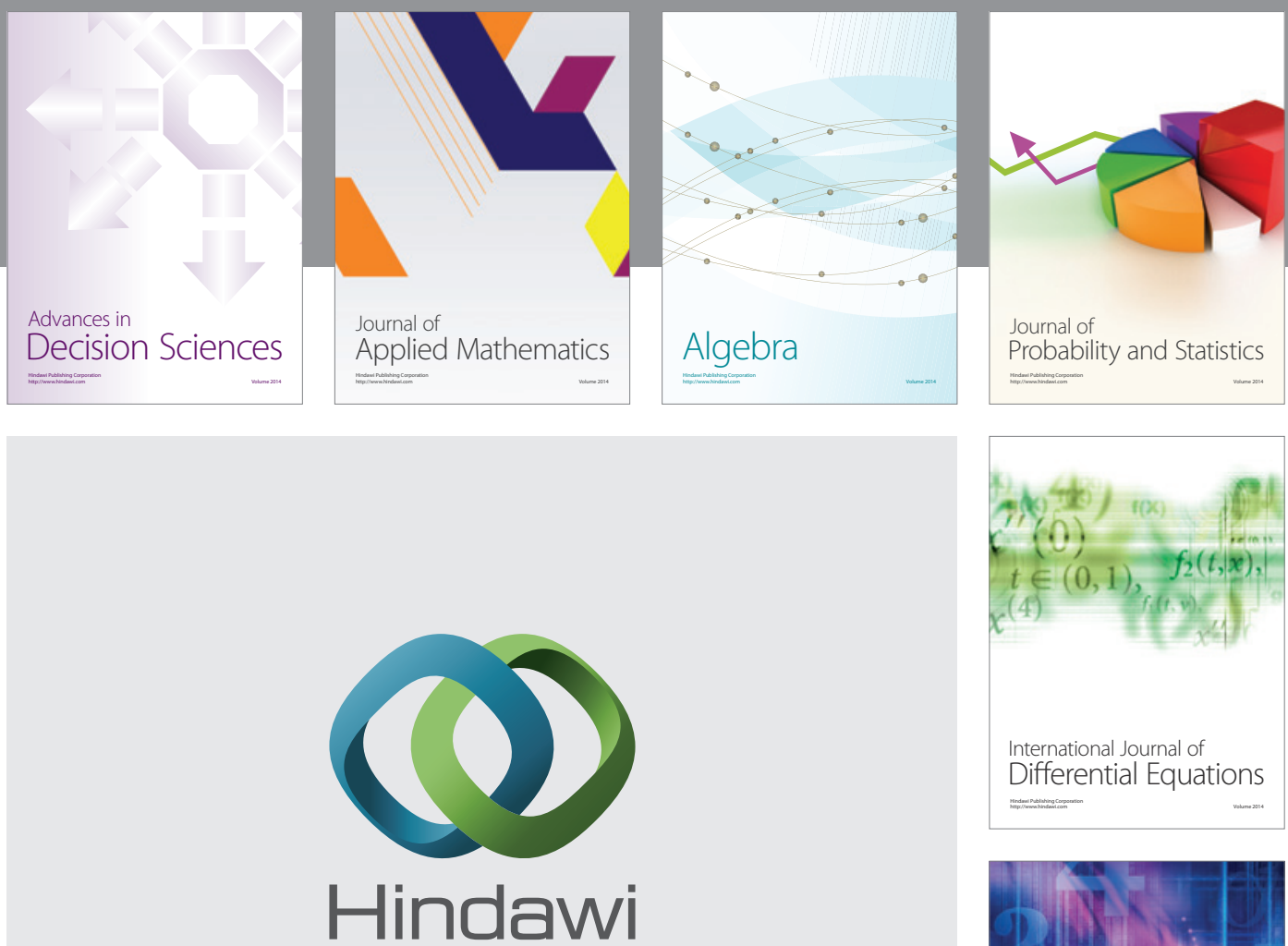

Submit your manuscripts at http://www.hindawi.com
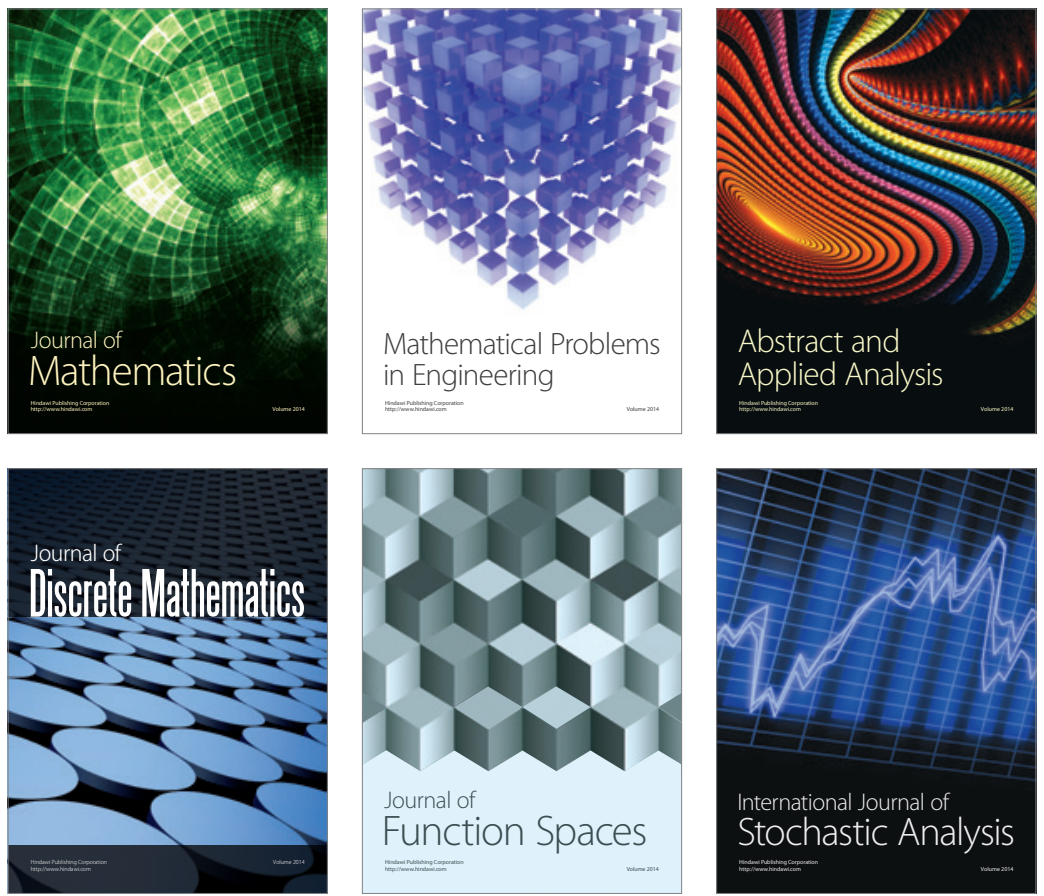

Journal of

Function Spaces

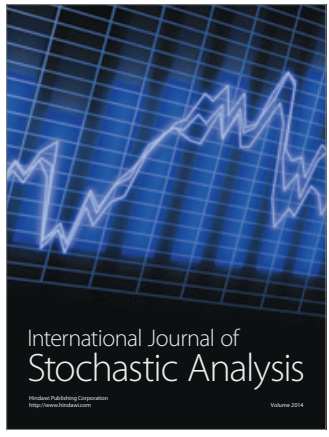

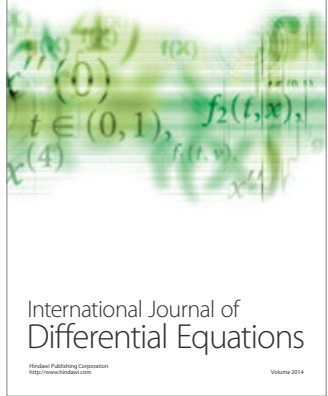
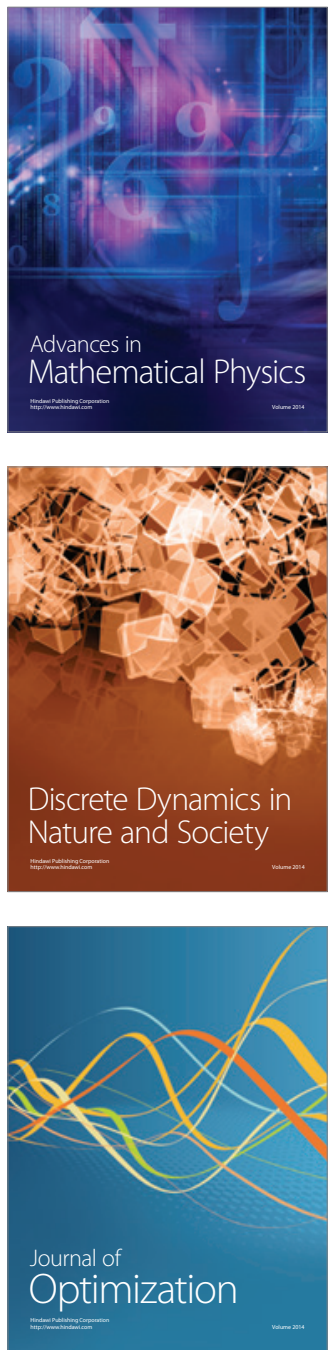